\title{
Overdose Education and Naloxone Distribution in the San Francisco County Jail
}

Journal of Correctional Health Care 2019, Vol. 25(4) 394-404

(C) The Author(s) 2019

Article reuse guidelines: sagepub.com/journals-permissions DOI: | 0.1 | $77 / 10783458|988277|$

journals.sagepub.com/home/jcx

\author{
Lynn D. Wenger, MSW, MPH', David Showalter, MA², \\ Barrot Lambdin, PhD', David Leiva ${ }^{3}$, Eliza Wheeler, MPA, MS ${ }^{4}$, \\ Peter J. Davidson, PhD $^{5}$, Phillip O. Coffin, MD ${ }^{6}$, \\ Ingrid A. Binswanger, MD $^{7}$, and Alex H. Kral, PhD'
}

\begin{abstract}
People leaving incarceration are at high risk of opioid-related overdose. Overdose fatalities are preventable with administration of naloxone. In response to this risk, overdose education and naloxone distribution (OEND) programs have been implemented in a handful of jails and prisons in the United States. We document the history, structure, and data from the San Francisco County Jail OEND program. During 4 years of operation, 637 people participated; $67 \%$ received naloxone upon release, of whom only $3.5 \%$ had been previously trained in community-based OEND programs. Of those who received naloxone, $32 \%$ reported reversing an overdose and $44 \%$ received refills from community-based programs after reentry. This confirms that implementation of OEND in criminal justice settings is feasible and reaches people who have not previously been trained as well as those willing to act as overdose responders.
\end{abstract}

\section{Keywords}

overdose prevention, naloxone distribution, criminal justice programming

Opioid overdose-related fatalities in the United States have increased dramatically over the last two decades. Drug overdose deaths nearly tripled between 2000 and 2014 (Rudd, Seth, David, \& Scholl, 2016). Recent studies have shown opioid overdose mortality continues to rise unabated. In 2016, opioid overdoses accounted for 53,332 deaths (National Institute on Drug Abuse, 2017), an increase

\footnotetext{
I RTI International, San Francisco, CA, USA

${ }^{2}$ Sociology Department, University of California, Berkeley, CA, USA

${ }^{3}$ San Francisco Department of Public Health, Jail Health Services, CA, USA

${ }^{4}$ Harm Reduction Coalition, Oakland, CA, USA

${ }^{5}$ University of California, San Diego, CA, USA

${ }^{6}$ Population Health Division, San Francisco Department of Public Health, Community Health Equity \& Promotion Branch, San Francisco, CA, USA

${ }^{7}$ Kaiser Permanente Colorado, Institute for Health Research, Denver, CO, USA
}

\section{Corresponding Author:}

Lynn D. Wenger, MSW, MPH, RTI International, 35I California St., Suite \#500, San Francisco, CA 94I04, USA.

Email: lynndee@rti.org 
from 33,091 in 2015 (Rudd et al., 2016). Adults aged 25 to 54 had the highest rates of drug overdose deaths (Hedegaard, Warner, \& Miniño, 2017), and overdose deaths have contributed to lowered life expectancy in the United States (Dowell et al., 2017).

People exiting incarceration are at high risk of overdose (Merrall et al., 2010). Binswanger and colleagues (2007) reported that people released from Washington State prisons had 129 times the risk of overdose death during the 2 weeks immediately following release than the general population. Opioids are the most common substance involved in fatal overdoses after release from incarceration (Alex et al., 2017; Binswanger, Blatchford, Mueller, \& Stern, 2013). Individuals recently released from jail or prison are at risk of opioid-related overdose likely due to reduced opioid tolerance while not using opioids when incarcerated and limited access to substance use disorder treatment and other services that support the transition from incarceration to community (Alex et al., 2017; Binswanger et al., 2007; Lim et al., 2012). Offering and providing comprehensive medicationassisted treatment (MAT) to people who use opioids in prisons and jails is one approach to reducing postrelease opioid overdose risk (Green et al., 2018). Despite evidence of improved outcomes, many jails and prisons do not offer MAT. The scarcity of MAT in jails and prisons results in poor outcomes for individuals and poses a public health threat to communities (Bone, Eysenbach, Bell, \& Barry, 2018).

Fatalities and other medical complications of opioid overdose are preventable with the timely administration of naloxone, an opioid antagonist. Historically, naloxone has been stocked by medical facilities that administer opioids and is standard medication on ambulances to be administered in the event of an opioid overdose (Davis, Southwell, Niehaus, Walley, \& Dailey, 2014). Naloxone is not a controlled substance and has no abuse potential.

In response to the growing opioid overdose epidemic, nearly all states have waived the requirement that naloxone be prescribed for use on a person with whom the provider has a traditional prescriber-patient relationship and have provided legal protection for naloxone prescribing, dispensing, and administration (Davis \& Carr, 2017). In this context, administration by laypersons is becoming more common (Wheeler, Jones, Gilbert, \& Davidson, 2015). Most naloxone administered by laypersons is obtained as part of community-based overdose education and naloxone distribution (OEND) programs, most often at syringe services programs (Doyon, Aks, \& Schaeffer, 2014). Providing OEND to laypersons who are likely to be bystanders in an overdose event, including people who use drugs, their family members, community members, and service providers, has been shown to be feasible, cost-effective, and associated with reduced opioid overdose fatalities (Bagley, Forman, Ruiz, Cranston, \& Walley, 2017; Coffin \& Sullivan, 2013; Doe-Simkins et al., 2014; Enteen et al., 2010; Sherman et al., 2008; Siegler et al., 2017; Sporer \& Kral, 2007; Tobin, Sherman, Beilenson, Welsh, \& Latkin, 2009; Wagner et al., 2010). Between 1996 and 2014, OEND programs in the United States distributed naloxone kits to over 152,000 laypersons and reported over 26,000 overdose reversals (Wheeler et al., 2015).

Prison- and jail-based OEND programs have been introduced in varying degrees in countries including Estonia, Spain, Norway, parts of the United Kingdom, Canada, and the United States (Horton et al., 2017). Although these programs exist worldwide, research about prerelease OEND programs is sparse in the public health literature. Results from an evaluation of an OEND program in Wales that provides trainings in community-based settings and prison locations noted an increase in participants' knowledge of how to recognize and respond to an overdose, their perceived confidence in responding to an overdose, and their willingness to carry out the recommended procedures to reverse an overdose after they took part in the program. In addition, participants who received naloxone demonstrated they were able to use naloxone in overdose events (Bennett \& Holloway, 2012). The OEND program in Scotland has been associated with a $36 \%$ reduction in the proportion of opioid-related overdoses that occurred in the 4 weeks following release from prison (Bird, McAuley, Perry, \& Hunter, 2016; Horsburgh \& McAuley, 2018). OEND training is also being 
offered to visitors at the Rikers Island jails in New York City. An evaluation of this program found that at 6-month follow-up, $14 \%$ of study participants had witnessed at least one overdose and $10 \%$ had administered naloxone at least once, confirming that offering OEND training in jail visitor centers is yet another mechanism to reach a population at risk of opioid-related overdose (HuxleyReicher et al., 2018).

In light of this evidence and in recognition of the elevated overdose risk experienced by people exiting incarceration in the United States, jurisdictions in multiple states (including California, Illinois, Maryland, Michigan, and New York) have implemented OEND programs in local jails and state prisons (Bathje \& Junge, 2016; Haas \& Forseth, 2016; White, Stancliff, \& Breslin, 2016; Zucker, Annucci, Stancliff, \& Catania, 2015). Although recruitment procedures, staff roles, and naloxone distribution policies vary across programs and states, the basic principles of OEND training curricula are similar. In this article, we provide descriptive and programmatic data on the OEND program in the San Francisco County Jail, which has been operating for 4 years, and make recommendations for implementation of OEND programs for people leaving incarceration.

\section{Program History}

The San Francisco County Jail OEND program aims to integrate overdose prevention into the wider array of services for substance-using adults in the jail and to decrease overdose mortality among people leaving incarceration and reentering the community. To do so, the program educates participants about overdose risks, teaches them the skills necessary to reverse an opioid overdose, and offers them the option of receiving naloxone in their property upon release.

The program is a collaboration among several county agencies and community partners. Within the jail, San Francisco County Jail Health Services (JHS) provides an integrated system of medical, psychiatric, and substance use disorder care including chronic care clinics; psychiatric care; jail aftercare services focusing on reentry planning for people with mental health and major medical needs; HIV prevention, testing, treatment, and case management; dental services; women's health care services; pharmacy services; and screening for tuberculosis, sexually transmitted infections, and hepatitis C virus (HCV; City and County of San Francisco, 2012). The JHS staff members who conduct OEND trainings work for a program providing HIV and HCV testing. Team members already have jail security clearance, relationships with correctional officers and other jail staff, and experience working with people who are incarcerated. JHS staff members are trained to provide OEND services by the Harm Reduction Coalition's Drug Overdose Prevention and Education (DOPE) project, which coordinates community-based distribution of naloxone in homeless shelters, substance use treatment programs, single room occupancy hotels, and syringe access programs in San Francisco. The DOPE project also pays for the naloxone and assembles the naloxone kits for the jail OEND program. Both JHS and the DOPE project are funded by the San Francisco Department of Public Health, whose medical director signed a standing order authorizing the DOPE project to provide OEND services. The jail OEND program began in a housing unit managed by San Francisco Adult Probation that provides a suite of reentry services. The San Francisco Sheriff's Department gives JHS staff access to jail housing and discharge records, allows JHS to use space within the jail to conduct trainings, and permits staff to put naloxone kits into program participants' property that is returned to them upon release from jail but has a minimal role in the implementation, funding, or ongoing operations of this program.

The San Francisco County Jail OEND program was established in 2013 as a pilot program operating in one male housing unit. In 2014, it began providing services in a female housing unit. The pilot program began as a supplement to JHS's HIV testing and disclosure program, with the goal of providing overdose prevention training to people within 30 days prior to their release date. In July 
2016, JHS received funding from the City of San Francisco to hire a dedicated overdose prevention staff person and expand the program to reach more people.

\section{OEND Program Model}

\section{Setting}

The San Francisco County Jail system is made up of five individual jails, two locked hospital units at San Francisco General Hospital providing inpatient physical and mental health care for people who are incarcerated, and a classification unit. The estimated population of the City and County of San Francisco was 884,363 in 2017 (U.S. Census Bureau, 2017); the average daily population of the jail system is approximately 1,535 , with an average length of stay of 4 months. The jail population is 91\% male and 9\% female (City and County of San Francisco, 2012). The San Francisco County Jail OEND program is housed in County Jail \#2, a direct supervision facility that utilizes pod architecture housing. County Jail \#2 houses men and women and is the system's only jail where women are housed (City and County of San Francisco Sheriff's Department, 2017). County Jail \#2 also has reentry housing units for individuals who are serving the last 30 to 90 days of their sentence. The reentry housing units provide programs such as cognitive behavioral programs, substance use treatment, education, parenting classes, case management, and linkages to community services. OEND programming fit well into the continuum of care provided in reentry housing units.

\section{Eligibility and Recruitment}

JHS staff are trained to provide OEND by trainers from the DOPE project using the same curriculum provided to service providers and community members in other venues in San Francisco. All people living in the reentry housing units who are within 30 days of their release date and who have not previously participated in the jail OEND program are eligible to participate. This includes people who use opioids and are personally at risk of overdose as well as those who have peers who use opioids. Once a month, JHS staff visit the reentry housing units and review the jail housing database to determine who is eligible to participate in the program. Eligible participants are called out of their cells by correctional officers to attend a group training conducted in a small classroom in their housing unit.

Since the addition of the overdose prevention staff position in 2016, the capacity of the OEND program has grown. Not only do the JHS staff members systematically review the reentry housing unit database to determine program eligibility, they also review intake records from all five county jails, looking for people who report opioid use prior to incarceration; these people are then flagged for OEND training. In addition, the jail medical staff can make direct referrals of people who they deem at risk of overdose upon release.

\section{OEND Training Curriculum}

Individuals who are interested and eligible first view a 19-minute video called "Staying Alive on the Outside," which is geared toward people approaching release from incarceration, with a focus on postrelease overdose prevention. The video was developed with input from people who use opioids, individuals with prior incarceration, correctional staff, and overdose prevention experts (Green et al., 2014). The video features previously incarcerated men and women as well as people who have lost family members to opioid overdose. It reviews the risks of postrelease overdose, advises how to reduce those risks, and teaches the viewer how to respond in the event of an overdose by performing rescue breathing and administering naloxone. The JHS staff person then briefly reviews overdose risk and prevention and answers questions. The staff person explains California's "Good 
Samaritan" law that provides some legal protections to people who call 911 or intervene to prevent overdose deaths and instructs people to call 911 as soon as possible.

People who are identified during queries of their intake records and those who are referred by medical staff receive a brief one-on-one training in a private setting (rather than as part of a group) in which the counselor reviews all elements of the video and provides detailed instructions on how to administer naloxone.

\section{Receiving Naloxone}

All OEND training participants are asked to fill out a slip of paper indicating whether they would like JHS staff to put a naloxone kit into their personal property, which they would receive upon release. Immediately following the training, those who opt in to receive the kit meet with JHS staff individually to review how to administer naloxone and to ask any questions.

The kit contains two doses of intranasal naloxone (NARCAN ${ }^{\circledR}$ Nasal spray 4 mgs), a naloxone nasal spray Quick Guide from the drug manufacturer, a San Francisco Department of Public Health (SFDPH/DOPE) project prescription card certifying that the person has been trained in overdose prevention and given a prescription to carry naloxone, referral information for when and where to get naloxone refills, an overdose prevention brochure designed by the DOPE Project, a schedule of syringe access sites in San Francisco, and a card with information about California's Good Samaritan law.

\section{Programmatic Data Collection}

All participants who ask to receive naloxone fill out a short survey with JHS staff. Data collected are programmatic data primarily used to generate reports to funding agencies and program administration. The survey questions include demographics, drug use 30 days prior to incarceration, prior experiences witnessing or experiencing overdose, and previous experience administering naloxone. When individuals receive a naloxone refill at a DOPE project community-based site, they fill out another short survey that includes their reason for obtaining a refill. If the reason is that the person used their naloxone to reverse an opioid overdose, they are asked specific questions about the overdose such as location, relationship to overdose victim, and outcome of overdose.

Registration and refill forms are collected from JHS staff by the DOPE project monthly and entered into the program's secure database by DOPE project staff. Data are entered using an encrypted connection to the server through a dedicated web interface (LimeSurvey, 2018).

\section{Programmatic Data}

Between March 2013 and April 2017, 637 program participants watched the OEND training video and $427(67 \%)$ opted to receiving naloxone in their property. An additional 26 people received oneon-one OEND training from JHS staff, all of whom received naloxone in their property.

Of the total 453 people who received naloxone during this time period, $50 \%$ were cisgender women, $48 \%$ were cisgender men, and $2 \%$ were transgender women; $40 \%$ were 30 to 40 years old; and $48 \%$ were African American, 30\% White, 17\% Latino, 7\% Native American, and 3\% other races or ethnicities (Table 1). In the 30 days prior to their incarceration, $33 \%$ had used heroin, $25 \%$ had used alcohol, $10 \%$ had used prescription opioids, $8 \%$ had used benzodiazepines, $6 \%$ had used methadone, and $2 \%$ had used buprenorphine. Sixteen participants $(3.5 \%)$ reported having participated in an OEND training prior to this incarceration.

Twenty-five percent $(n=112)$ of program participants who received naloxone in their property had experienced an opioid overdose at least once in their lifetime, and $47 \%(n=53)$ of those who 
Table I. Demographic Characteristics, Drug Use, and Overdose Experience of Program Participants Who Received Naloxone Kit Upon Release From San Francisco County Jail.

\begin{tabular}{|c|c|c|}
\hline Demographics & $N$ & $\%$ \\
\hline \multicolumn{3}{|l|}{ Gender } \\
\hline Cisgender women & 226 & 50 \\
\hline Cisgender men & 216 & 48 \\
\hline Transgender women & II & 2 \\
\hline \multicolumn{3}{|l|}{ Age } \\
\hline$<30$ years old & 128 & 28 \\
\hline $30-40$ years old & 179 & 40 \\
\hline$>40$ years old & 146 & 32 \\
\hline \multicolumn{3}{|l|}{ Ethnicity } \\
\hline African American & 218 & 48 \\
\hline White & 137 & 30 \\
\hline Latino & 77 & 17 \\
\hline Asian & 30 & 7 \\
\hline Native American & 25 & 6 \\
\hline Other & 15 & 3 \\
\hline \multicolumn{3}{|l|}{ Drug use 30 days prior to arrest } \\
\hline None & 70 & 15 \\
\hline Heroin & 149 & 33 \\
\hline Methadone & 27 & 6 \\
\hline Buprenorphine or suboxone & 10 & 2 \\
\hline Prescription opioids & 45 & 10 \\
\hline Benzodiazepines & 35 & 8 \\
\hline Cocaine or crack & 110 & 24 \\
\hline Methamphetamines & 199 & 44 \\
\hline Alcohol & 112 & 25 \\
\hline
\end{tabular}

Note. $N=453$.

Table 2. Experiences With Overdose, Naloxone, and Naloxone Refills of Program Participants Who Received Postrelease Naloxone.

\begin{tabular}{lcc}
\hline Overdose/Naloxone Experiences & $N$ & $\%$ \\
\hline Ever overdosed & 112 & 25 \\
Ever been revived with naloxone: & $53 / 112$ & 47 \\
$\quad$ Revived by bystander (friend, partner, etc.) & $27 / 53$ & 51 \\
Revived by medical personnel or first responder & $30 / 53$ & 57 \\
Ever witnessed an overdose & 286 & 63 \\
At last overdose did anyone including participant administer naloxone & $111 / 286$ & 39 \\
Reason for getting naloxone upon release: & 127 & 28 \\
I am at risk & 393 & 87 \\
My friends, partner, neighbor, or people in my community are at risk & 116 & 26 \\
I have a family member at risk & 2 & $<1$ \\
I work in an agency with people who are at risk & & \\
\hline
\end{tabular}

Note. $N=453$.

had experienced an opioid overdose had been revived with naloxone at least once, either by a bystander or a first responder. Sixty-three percent $(n=286)$ reported having witnessed at least one overdose in their lifetime. Of those 286 participants, $39 \%(n=112)$ stated that either they or 
someone else had administered naloxone at the time of the last witnessed overdose. When asked why they wanted to receive naloxone upon release, $87 \%(n=393)$ responded that their friends, partner, neighbor, or people in their community were at risk of overdose. (Table 2)

Of the participants who received naloxone in their property, 198 (44\%) later received a naloxone refill in the community: $190(96 \%)$ of these individuals received their refill at a syringe access program or other community-based program in San Francisco that provides OEND, and 8 (4\%) received their refill at the jail during a subsequent incarceration. The most frequent reasons reported for getting a naloxone refill were that the naloxone was lost $(33 \%)$, had been used to reverse an overdose (32\%), had been stolen (13\%), and had been given away to someone who needed it, who for some reason could not get it themselves (12\%).

\section{Discussion}

Implementation of OEND in correctional facilities addresses the needs of persons leaving incarceration who are at very high risk of experiencing or witnessing opioid overdose. Programs are being implemented in several jurisdictions across the country using similar educational curricula with varying programmatic structures. Programmatic data from the San Francisco County Jail OEND program show that implementation of these types of programs within criminal justice settings is feasible.

Several features facilitated the implementation of OEND in the San Francisco County Jail. The SFDPH medical director had written a standing order to allow naloxone to be distributed throughout the City and County of San Francisco, including the County Jail. San Francisco had an established harm reduction program, the DOPE project, that provided OEND trainings in multiple community settings across the city, which initiated and led the process to negotiate staffing, training, and funding for the jail program. JHS staff had already obtained jail clearance and were providing HIV and HCV testing services in the jail to people who use drugs; implementation of OEND allowed them to provide a lifesaving public health intervention that aligned with their existing services and mission.

The OEND program in the San Francisco jail reaches people who are incarcerated and are personally at risk of opioid overdose or who live in communities where people are at risk of overdose. Correctional education programs are intended to provide people who are incarcerated with the skills they need to succeed postrelease. Premised on the belief that rehabilitation through education is more effective and humane than the threat of further punishment, correctional education programs have been credited with reducing recidivism among program participants and reducing disciplinary infractions among incarcerated populations (Klein, Tolbert, Bugarin, Cataldi, \& Tasuchek, 2004). Given that most San Francisco County Jail OEND program participants who received naloxone had witnessed an opioid-related overdose and many had personal experience with overdose, they were highly motivated to participate in this brief educational program. It is important to note that the program also reached at-risk persons who had not previously accessed OEND in the community.

In addition to preventing fatal overdoses, participation in OEND programs helps individuals minimize drug-related harm and encourages them to take on new prosocial roles in their community as peer educators and "overdose responders" (Seal et al., 2005; Wagner et al., 2014). After receiving training, participants often teach others in their communities about overdose risk and response. Through this process of information diffusion and skill sharing, participants provide a service to their community and broaden the reach of OEND programs to individuals who do not attend formal community-based trainings (Sherman et al., 2009). Participants report feelings of heroism, confidence, satisfaction, increased self-esteem, and improved self-worth associated with their new role (Wagner et al., 2014). Using naloxone to reverse overdoses earns participants' approval from peers and professionals, helping to develop and reaffirm positive identities as caretakers and experts and to 
reject negative stereotypes often applied to people who use drugs (Wagner et al., 2014). This increased sense of self-worth, responsibility for others, and relations of reciprocal trust and care are factors that are conducive to reintegration with the community (Maruna, 2001; National Research Council, 2008; Sampson \& Laub, 1993).

There are several limitations to this program evaluation, including that all data collected are selfreported and may be subject to social desirability and recall bias. In addition, the data were collected primarily for programmatic purposes. Despite these limitations, the finding that approximately $97 \%$ of OEND participants had never been trained before, even though they are in one of the most saturated OEND markets in the United States, indicates that this program is reaching a segment of the population that is not accessing services in the community. Another notable finding is that more than $40 \%$ of participants who went through OEND training in the San Francisco jail obtained a refill in the community and $32 \%$ of those who requested a refill used their naloxone to reverse an overdose. Although requesting a refill is likely an underestimation of naloxone administration and overdose reversal, it does show that this program is targeting a population that needs this intervention and who are willing and able to use the skills and tools within the community.

In conclusion, programs such as the OEND program in the San Francisco County Jail are reaching individuals at elevated risk of opioid overdose as well as those who are reentering communities where they may witness an opioid-related overdose. This program and others like it are educating incarcerated individuals about their own risk of overdose upon release from correctional facilities as well as training them to become overdose responders in their communities. OEND training prior to release from jail supports the reentry process by providing participants with skills that make them valued and valuable members of their communities. Along with similar programs that have been successfully implemented in Europe, this U.S. program establishes the feasibility of naloxone upon release from incarceration (Horton et al., 2017). Future research should investigate efforts to develop these programs in a variety of locations and correctional settings in the United States to uncover barriers and facilitators to effective implementation.

\section{Declaration of Conflicting Interests}

The authors declared no potential conflicts of interest with respect to the research, authorship, and/or publication of this article. For information about $J C H C$ 's disclosure policy, please see the Self-Study Exam

\section{Funding}

The authors disclosed receipt of the following financial support for the research, authorship, and/or publication of this article: This study received funding from the National Institutes on Drug Abuse (grant ID 5R34DA039101-03).

\section{References}

Alex, B., Weiss, D. B., Kaba, F., Rosner, Z., Lee, D., Lim, S., . . MacDonald, R. (2017). Death after jail release. Journal of Correctional Health Care, 23, 83-87. doi:10.1177/1078345816685311

Bagley, S. M., Forman, L. S., Ruiz, S., Cranston, K., \& Walley, A. Y. (2017). Expanding access to naloxone for family members: The Massachusetts experience. Drug and Alcohol Review, 37, 480-486. doi:10.1111/dar. 12551

Bathje, G., \& Junge, E. (2016, November). Overdose prevention within law enforcement and incarceration systems. In D. McCord (Moderator), Overdose in the criminal justice system. Panel conducted at the 11th National Harm Reduction Conference, San Diego, CA.

Bennett, T., \& Holloway, K. (2012). The impact of take-home naloxone distribution and training on opiate overdose knowledge and response: An evaluation of the THN project in wales. Drugs: Education, Prevention and Policy, 19, 320-328. 
Binswanger, I. A., Blatchford, P. J., Mueller, S. R., \& Stern, M. F. (2013). Mortality after prison release: Opioid overdose and other causes of death, risk factors, and time trends from 1999 to 2009. Annals of Internal Medicine, 159, 592-600. doi:10.7326/0003-4819-159-9-201311050-00005

Binswanger, I. A., Stern, M. F., Deyo, R. A., Heagerty, P. J., Cheadle, A., Elmore, J. G., \& Koepsell, T. D. (2007). Release from prison-A high risk of death for former inmates. New England Journal of Medicine, 356, 157-165. doi:10.1056/NEJMsa064115

Bird, S. M., McAuley, A., Perry, S., \& Hunter, C. (2016). Effectiveness of Scotland's National Naloxone Programme for reducing opioid-related deaths: A before (2006-10) versus after (2011-13) comparison. Addiction, 111, 883-891. doi:10.1111/add.13265

Bone, C., Eysenbach, L., Bell, K., \& Barry, D. T. (2018). Our ethical obligation to treat opioid use disorder in prisons: A patient and physician's perspective. Journal of Law, Medicine \& Ethics, 46, 268-271. doi:10. $1177 / 1073110518782933$

City and County of San Francisco. (2012). San Francisco Department of Public Health Jail Health Services: Overview of services FY 11-12. Retrieved from https://www.sfdph.org/dph/hc/Orientation\%202013/11$12 \% 20$ Overview\%20jail.pdf

City and County of San Francisco Sheriff's Department. (2017). San Francisco county jail system facility descriptions/ county jail \#2. Retrieved from http://www.sfsheriff.com/jail_info.html

Coffin, P. O., \& Sullivan, S. D. (2013). Cost-effectiveness of distributing naloxone to heroin users for lay overdose reversal in Russian cities. Journal of Medical Economics, 16, 1051-1060. doi:10.3111/13696998. 2013.811080

Davis, C. S., \& Carr, D. (2017). State legal innovations to encourage naloxone dispensing. Journal of the American Pharmacists Association, 57, S180-S184.

Davis, C. S., Southwell, J. K., Niehaus, V. R., Walley, A. Y., \& Dailey, M. W. (2014). Emergency medical services naloxone access: A national systematic legal review. Academic Emergency Medicine, 21, 1173-1177. doi:10.1111/acem.12485

Doe-Simkins, M., Quinn, E., Xuan, Z., Sorensen-Alawad, A., Hackman, H., Ozonoff, A., \& Walley, A. Y. (2014). Overdose rescues by trained and untrained participants and change in opioid use among substanceusing participants in overdose education and naloxone distribution programs: A retrospective cohort study. BMC Public Health, 14, 297. doi:10.1186/1471-2458-14-297

Dowell, D., Arias, E., Kochanek, K., Anderson, R., Guy, G. P. Jr., Losby, J. L., \& Baldwin, G. (2017). Contribution of opioid-involved poisoning to the change in life expectancy in the United States, 20002015. Journal of the American Medical Association, 318, 1065-1067. doi:10.1001/jama.2017.9308

Doyon, S., Aks, S. E., \& Schaeffer, S. (2014). Expanding access to naloxone in the United States. Journal of Medical Toxicology, 10, 431-434. doi:10.1007/s13181-014-0432-1

Enteen, L., Bauer, J., McLean, R., Wheeler, E., Huriaux, E., Kral, A. H., \& Bamberger, J. D. (2010). Overdose prevention and naloxone prescription for opioid users in San Francisco. Journal of Urban Health, 87, 931-941. doi:10.1007/s11524-010-9495-8

Green, T. C., Bowman, S. E., Ray, M., McKenzie, M., Lord, S. E., \& Rich, J. D. (2014). Development of an incarceration-specific overdose prevention video: "Staying Alive on the Outside". Health Education Journal, 74, 627-637.

Green, T. C., Clarke, J., Brinkley-Rubinstein, L., Marshall, B. D. L., Alexander-Scott, N., Boss, R., \& Rich, J. D. (2018). Postincarceration fatal overdoses after implementing medications for addiction treatment in a statewide correctional system. Journal of the American Medical Association Psychiatry, 75, 405-407. doi: 10.1001/jamapsychiatry.2017.4614

Haas, E., \& Forseth, K. (2016, November). Overdose education and naloxone delivery in local detention centers in Maryland. In D. McCord (Moderator), Overdose in the criminal justice system. Panel conducted at the 11th National Harm Reduction Conference, San Diego, CA.

Hedegaard, H., Warner, M., \& Miniño, A. M. (2017). Drug overdose deaths in the United States, 1999-2016 (NCHS Data Brief no. 294). Hyattsville, MD: National Center for Health Statistics. 
Horsburgh, K., \& McAuley, A. (2018). Scotland's National Naloxone Program: The prison experience. Drug and Alcohol Review, 37, 454-456. doi:10.1111/dar.12542

Horton, M., McDonald, R., Green, T. C., Nielsen, S., Strang, J., Degenhardt, L., \& Larney, S. (2017). A mapping review of take-home naloxone for people released from correctional settings. International Journal on Drug Policy, 46, 7-16. doi:10.1016/j.drugpo.2017.05.015

Huxley-Reicher, Z., Maldjian, L., Winkelstein, E., Siegler, A., Paone, D., Tuazon, E., . . Kunins, H. V. (2018). Witnessed overdoses and naloxone use among visitors to Rikers Island jails trained in overdose rescue. Addictive Behaviors, 86, 72-78. doi:10.1016/j.addbeh.2017.11.029

Klein, S., Tolbert, M., Bugarin, R., Cataldi, E. F., \& Tauschek, G. (2004). Correctional education: Assessing the status of prison programs and information needs. Washington, DC: Office of Safe and Drug-Free Schools, U.S. Department of Education. Retrieved from https://files.eric.ed.gov/fulltext/ED543577.pdf

Lim, S., Seligson, A. L., Parvez, F. M., Luther, C. W., Mavinkurve, M. P., Binswanger, I. A., \& Kerker, B. D. (2012). Risks of drug-related death, suicide, and homicide during the immediate post-release period among people released from New York City jails, 2001-2005. American Journal of Epidemiology, 175, 519-526. doi:10.1093/aje/kwr327

LimeSurvey GmbH. (2018). Professional online surveys with LimeSurvey. Retrieved from https://www.lime survey.org/

Maruna, S. (2001). Making good: How ex-convicts reform and rebuild their lives. Washington, DC: American Psychological Association.

Merrall, E. L., Kariminia, A., Binswanger, I. A., Hobbs, M. S., Farrell, M., Marsden, J., . . Bird, S. M. (2010). Meta-analysis of drug-related deaths soon after release from prison. Addiction, 105, 1545-1554. doi:10. $1111 /$ j.1360-0443.2010.02990.x

National Institute on Drug Abuse. (2017). Overdose death rates. Retrieved from https://www.drugabuse.gov/ related-topics/trends-statistics/overdose-death-rates

National Research Council. (2008). Parole, desistance from crime, and community integration. Washington, DC: National Academies Press.

Rudd, R. A., Seth, P., David, F., \& Scholl, L. (2016). Increases in drug and opioid-involved overdose deathsUnited States, 2010-2015. Morbidity and Mortality Weekly Report, 65, 1445-1452. doi:10.15585/mmwr. mm655051e1

Sampson, R. J., \& Laub, J. H. (1993). Crime in the making: Pathways and turning points through life. Cambridge, MA: Harvard University Press.

Seal, K. H., Thawley, R., Gee, L., Bamberger, J., Kral, A. H., Ciccarone, D., .. Edlin, B. R. (2005). Naloxone distribution and cardiopulmonary resuscitation training for injection drug users to prevent heroin overdose death: A pilot intervention study. Journal of Urban Health, 82, 303-311. doi:10.1093/jurban/jti053

Sherman, S. G., Gann, D. S., Scott, G., Carlberg, S., Bigg, D., \& Heimer, R. (2008). A qualitative study of overdose responses among Chicago IDUs. Harm Reduction Journal, 5, 2. doi:10.1186/1477-7517-5-2

Sherman, S. G., Gann, D. S., Tobin, K. E., Latkin, C. A., Welsh, C., \& Bielenson, P. (2009). "The life they save may be mine": Diffusion of overdose prevention information from a city sponsored programme. International Journal on Drug Policy, 20, 137-142. doi:10.1016/j.drugpo.2008.02.004

Siegler, A., Huxley-Reicher, Z., Maldjian, L., Jordan, R., Oliver, C., Jakubowski, A., \& Kunins, H. V. (2017). Naloxone use among overdose prevention trainees in New York City: A longitudinal cohort study. Drug and Alcohol Dependence, 179, 124-130. doi:10.1016/j.drugalcdep.2017.06.029

Sporer, K. A., \& Kral, A. H. (2007). Prescription naloxone: A novel approach to heroin overdose prevention. Annals of Emergency Medicine, 49, 172-177. doi:10.1016/j.annemergmed.2006.05.025

Tobin, K. E., Sherman, S. G., Beilenson, P., Welsh, C., \& Latkin, C. A. (2009). Evaluation of the staying alive programme: Training injection drug users to properly administer naloxone and save lives. International Journal on Drug Policy, 20, 131-136. doi:10.1016/j.drugpo.2008.03.002

U.S. Census Bureau. (2017). Quickfacts/San Francisco county, California. Retrieved from https://www.census. gov/quickfacts/fact/table/sanfranciscocountycalifornia/PST045217\#viewtop 
Wagner, K. D., Davidson, P. J., Iverson, E., Washburn, R., Burke, E., Kral, A. H., . . Lankenau, S. E. (2014). “I felt like a superhero": The experience of responding to drug overdose among individuals trained in overdose prevention. International Journal on Drug Policy, 25, 157-165. doi:10.1016/j.drugpo.2013.07.003

Wagner, K. D., Valente, T. W., Casanova, M., Partovi, S. M., Mendenhall, B. M., Hundley, J. H., ... Unger, J. B. (2010). Evaluation of an overdose prevention and response training programme for injection drug users in the Skid Row area of Los Angeles. CA. International Journal on Drug Policy, 21, 186-193. doi:10.1016/j. drugpo.2009.01.003

Wheeler, E., Jones, T. S., Gilbert, M. K., \& Davidson, P. J. (2015). Opioid overdose prevention programs providing naloxone to laypersons-United States, 2014. Morbidity and Mortality Weekly Report, 64, 631-635.

White, V., Stancliff, S., \& Breslin, D. (2016, November). Training incarcerated individuals prior to release and equipping them with naloxone upon release. In D. McCord (Moderator), Overdose in the criminal justice system. Panel conducted at the 11th National Harm Reduction Conference, San Diego, CA.

Zucker, H., Annucci, A. J., Stancliff, S., \& Catania, H. (2015). Overdose prevention for prisoners in New York: A novel program and collaboration. Harm Reduction Journal, 12, 51. doi:10.1186/s12954-015-0084-8 